\title{
Empirical study of the influence of social groups in evacuation scenarios
}

\author{
Cornelia von Krüchten, Frank Müller, Anton Svachiy, Oliver Wohak and Andreas \\ Schadschneider
}

\begin{abstract}
The effects of social groups on pedestrian dynamics, especially in evacuation scenarios, have attracted some interest recently. However, due to the lack of reliable empirical data, most of the studies focussed on modelling aspects. It was shown that social groups can have a considerable effect, e.g. on evacuation times. In order to test the model predictions we have performed laboratory experiments of evacuations with different types and sizes of the social groups. The experiments have been performed with pupils of different ages. Parameters that have been considered are (1) group size, (2) strength of intra-group interactions, and (3) composition of the groups (young adults, children, and mixtures). For all the experiments high-quality trajectories for all participants have been obtained using the PeTrack software. This allows for a detailed analysis of the group effects. One surprising observation is a decrease of the evacuation time with increasing group size.
\end{abstract}

\section{Introduction}

The influence of social groups in pedestrian dynamics, especially in evacuation scenarios, is an area of recent interest, see e.g. [1, 2] and other contributions in these proceedings. The situations that are considered are widespread and well-known in everyday life. For example, many people visit concerts or soccer matches not alone, but together with family and friends in so-called social groups. In case of emergency, these groups will try to stay together during an evacuation. The strength of this cohesion depends on the composition of the social group. Several adult friends

Cornelia von Krüchten · Frank Müller · Anton Svachiy · Oliver Wohak

Institut für Theoretische Physik, Universität zu Köln, 50937 Köln, e-mail: cvk@thp. uni-koeln.de

Andreas Schadschneider

Institut für Physik und ihre Didaktik and Institut für Theoretische Physik, Universität zu Köln, 50937 Köln, e-mail: as@thp.uni-koeln. de 
would form a loose group that is mainly connected via eye contact, whereas a mother would take her child's hand and form a strong or even fixed bond. In addition, even the size of the social groups could have an effect on the evacuation behaviour.

In order to consider these phenomena in a more detailed way, a cooperation of researchers of the universities of Cologne and Wuppertal and the Forschungszentrum Jülich has performed several experiments aiming at the determination of the general influence of inhomogeneities on pedestrian dynamics. They contained two series of experiments with pupils of different ages in two schools in Wuppertal. The first series focussed on the determination of the fundamental diagram of inhomogeneous groups, i.e. pedestrians of different size. The second series of experiments considered evacuation scenarios. In several runs the parameters of the crowd of evacuating pupils were varied, i.e. the size of the social group and its structure and the interaction between the group members. Here we present first results for these evacuation experiments.

\section{Teaching units}

The experiments were accompanied by teaching units for all involved students providing an introduction into the topic of traffic and pedestrian dynamics.

In classes of fifth and sixth grade, the focus of the classes was on the important quantities of pedestrian dynamics, especially density, time and bottleneck situations. This introduction to crowd effects and pedestrian behaviour was intended to raise awareness for their relevance for their everyday lives and safety issues. Therefore we arranged little experiments the students could perform themselves, e.g. the panic experiment according to Mintz [3] (see Fig. 11. In small groups the pupils had to pull several wooden wedges out of a bottle with a narrow neck as fast as possible and observe the blocking of the wedges when every students pulls at the same time. This experiment was supposed to indicate that coordination can lead to better results compared to selfish behavior.

The older pupils of classes 10 and 11 participated in an introduction to cellular automata and the physics of traffic. They received several worksheets on the Game of Life and other cellular automata, especially the Nagel-Schreckenberg model [4]. The aim of these lessons was to obtain a first qualitative and quantitative understanding of the collective effects in traffic systems. This should help to increase the identification with the experiments they later participated in and raise awareness about the relevance of this kind of research for everyday life. 
Fig. 1 Panic experiment according to Mintz. Every pupil is assigned a cord with a wedge on its end lying in a bottle with a narrow neck. If every student pulls at the same time and as fast as possible, the wedges block at the bottleneck. On the other hand, behaving in a coordinate way leads to a smooth process that is significantly faster. (Photo by V. Ziemer, U Wuppertal)

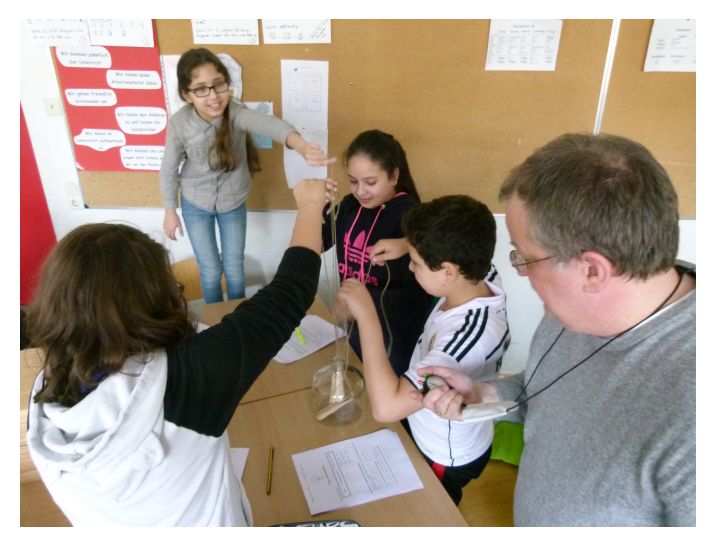

\section{Experimental set-up and procedure}

The experiments were performed in two schools in Wuppertal. Students of four classes participated as part of project work. The experimental room was built in the school's assembly hall.

\subsection{Experimental set-up}

The experimental area was a square room of $5 \times 5 \mathrm{~m}^{2}$ bounded by several small buckets. In the center of this area there was a square starting area of $3 \times 3 \mathrm{~m}^{2}$ denoted by the white marks. The students stepped into the room through the entrance that is shown below in Fig. 2 and assembled in the starting area. During the evacuation they had to leave the room using the exit on the left side. The exit door was built by two upstanding platforms and had a variable width changing between $0.8 \mathrm{~m}$ and $1.2 \mathrm{~m}$. The area behind the door was connected to the waiting area before the entrance, so the students could walk on a closed path. For the collection of data all experiments were recorded by a camera system. This system was mounted on the hall's ceiling and contained customary digital cameras and GoPros.

All students wore caps of different colour. Each colour represented a certain interval of body heights. The body height of each pupil was measured before the experiments started. This information is needed to determine the position accurately, but the different colours can also be used to draw conclusions about the composition of the group of evacuating pupils later in the video. All caps showed also a black point at the middle of the head. That allows to recognize and track each person in the video. 
Fig. 2 The experimental area consists of a square room of $5 \times 5 \mathrm{~m}^{2}$, with a starting area in the middle. The pupils had to leave this room through the exit at the left. They wore colourful caps to distinguish the different body heights.

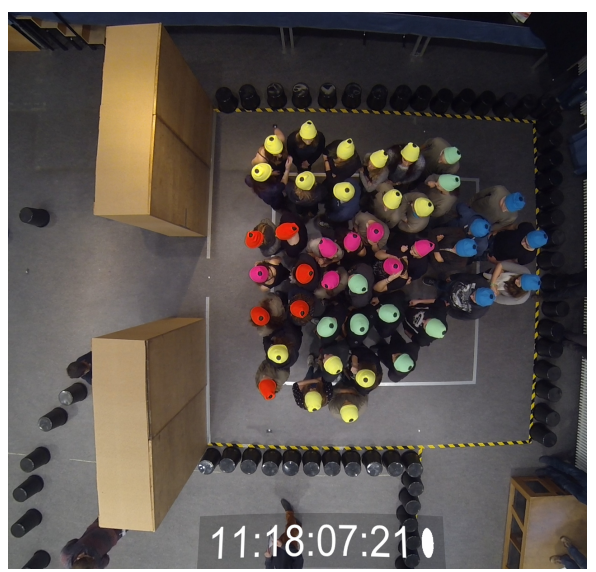

\subsection{Experimental procedure}

In general, the pupils had to perform several evacuation runs. For each run, a group of 32 to 46 persons assembled in the starting area, distributed nearly uniformly. During the evacuation, the students were allowed to use the whole experimental area.

After a starting signal, the participants had to leave the room using the exit door. They should walk briskly and evacuate the room as fast as possible. The pupils were told to imagine there would be a kind of danger, like fire or smoke. However, they were not allowed to run, scramble or push each other. After leaving the room they had to assemble again in the waiting area in front of the entrance and to wait for the next run.

The group of pupils that was placed into the experiment was compound in different ways to consider different parameters.

The first parameter that was varied in the experiment was the composition of the entire group. At all, there were two different age classes allowing for three different group compositions. The crowd could consist only of children aged 10 to 12 years, only of young adults aged 15 to 17 , or a mixture of both groups whereby children and youths were equally represented.

The second parameter concerned the social group size. In several runs, the students had either to evacuate on their own without regarding the others around them, or to form pairs, or larger social groups. These groups could contain four, six or eight persons. Within one pair or social group the students had to try to stay together during the evacuation run.

As a third parameter we considered the interaction within the social group. The nature of the interaction can be specified by (i) its strength and (ii) the hierarchy of group members. Regarding the interaction strength, the group members could either be connected loosely, by just trying to stay together via eye contact, or they could have a fixed bond. A fixed bond was realized by holding each other's hand 
or some other physical contact. Furthermore, hierarchy of the group members could be different. In the first case, all partners were treated equally. Each group member had to leave the room and to stay together with their partners. In the other case, one student was declared as the "leader", the other one as the "follower". The leader had to leave the room without regarding its partner or the other students, whereas the follower just had to follow the leader through the room.

This leads to four different ways to form pairs during the evacuation run. In the case of age-matched partners, the leader was chosen randomly. For the runs with the mixed crowd, the pairs were composed of one child and one teenager that took the part of the leader in the runs they were needed. All runs with larger social groups were done with loose bonds. In social groups of same age, there was no leader, but in mixed social groups one of the youths was declared as the leader.

To analyze the experiments the videos of the camera system that was mounted on the hall's ceiling was available. For each run of the experiment there is a video sequence. Using the PeTrack software [5], it is possible to extract the trajectories for each person and each run. The students were recognized via the black point in the middle of their coloured caps. The position of this point was tracked in each frame, generating the trajectory of each participant.

\section{Analysis}

First, we focus on the analysis of the data regarding the influence of group size on the evacuation scenario. Therefore, we use the data of one school and of the runs with larger groups. Most of these experiments were performed only with the older pupils with loose bonds and no leader-follower relationship, to which we restrict our analysis for now.

In different runs, the students formed groups of four, six and eight persons. In addition, one run with groups of six students and with an explicitly cooperative behaviour within the group was performed. They should concentrate a bit more on their group members and try to leave the room together. For comparison, we also consider the run with pairs and a loose bond that can be seen as a smaller group of two persons.

\subsection{Evacuation times for large groups}

First we consider the evacuation time. In Fig. 3, we plot the number of evacuated persons against the time needed to leave the room. The results can be compared between the different runs.

The evacuation time for each person is defined as the time difference between the beginning of the evacuation and the moment when the person passes the door, exactly when he/she leaves the aisle that is formed by the two platforms. The begin- 


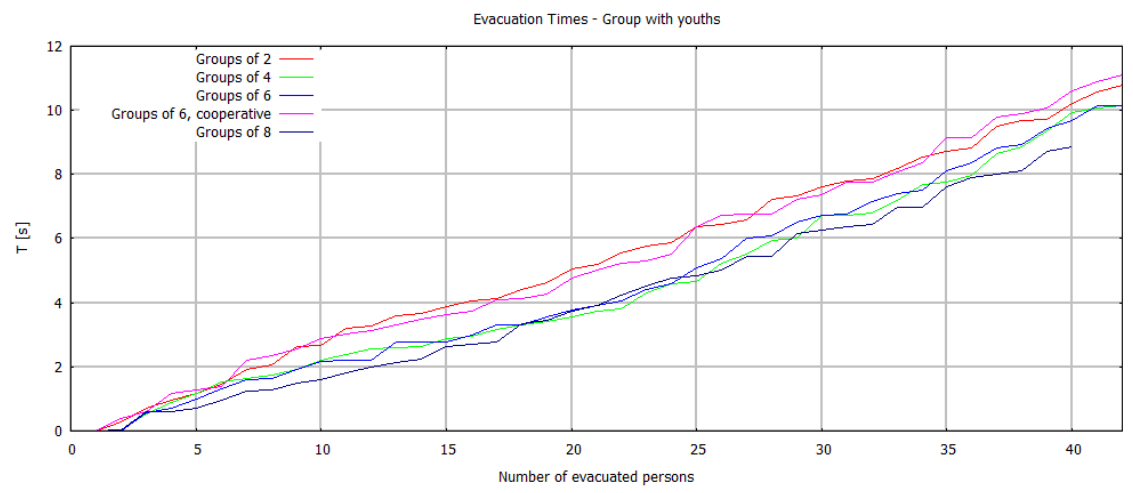

Fig. 3: Evacuation times for larger groups of one of the schools. The splitting of the curves into two groups is obvious. The shorter evacuation times belong to the runs with larger groups of four, six and eight persons, the longer ones to those with pairs and cooperative behaviour.

ning of the evacuation can be determined only approximately because the starting signal is not audible in the videos that are used for the extraction of the trajectories. For extracting the evacuation times we set the beginning on the moment of the first movement towards the door. However, for the analysis the influences of this inaccurate definition, the pre-movement time or other delays should be minimized. In doing so, we take the evacuation time of the very first person that left the room and subtract it from all the other times. Thereby, all plots start at zero for the first person and it is easier to compare different runs.

For the analysis of the runs with larger groups the evacuation times are shown in Fig. 3 All graphs show a nearly linear behaviour that could be expected. At the beginning of the evacuation all evacuation times are roughly the same. Between three and six evacuated persons the curves start to split into two groups. After increasing slightly, the difference between the two progresses remains nearly constant until the end of the evacuation. The main insight is that there are several runs that are clearly faster than other ones.

The upper two curves represent the evacuation in pairs and in groups of six with very cooperative behaviour. The lower graphs show the runs with larger groups of four, six and eight persons. Within the two groups of curves the differences are not large enough to separate the runs from each other. However, in the lower group, the run with eight participants per group seems to be a bit faster at the end of the evacuation. The run with six participants per group and cooperative behaviour is clearly slower than the run with same group size but without this instruction. These results indicate that forming groups is advantageous for the evacuation, whereas behaving cooperatively inhibits this effect.

While looking for reasons for the differences in evacuation times, one first approach could be to determine the density distribution. Therefore, we determined the 


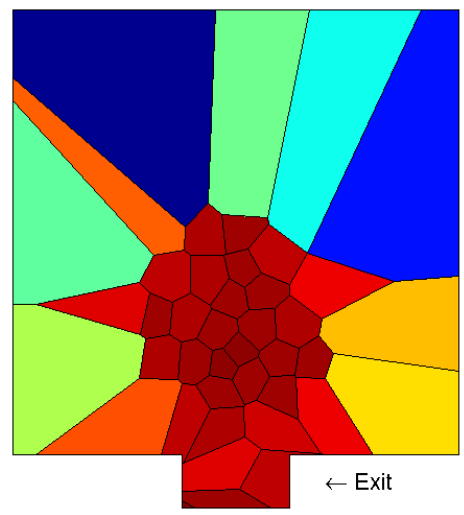

(a) Pairs at $4 \mathrm{~s}$

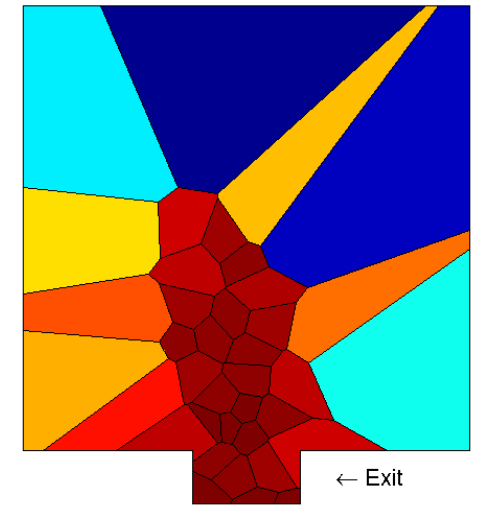

(b) Groups of four at $4 \mathrm{~s}$

Fig. 4: Voronoi cells for each person in the room at time $4 \mathrm{~s}$ for the runs with pairs and social groups of four persons. The colour of each cell depends on its size as a measure of density.

Voronoi cells [6, 7] within the experimental room for each person at different times. As a measure of density we coloured all cells dependent on their size: smaller cells are coloured in shades of red, larger ones in blue.

In Fig. 4 the density distributions for the run with pairs and with groups of four persons are shown. It is clearly seen that the distribution for the run in (b) is a bit narrower than the other one at the same time step. That means when forming groups, the children order rather behind each other than next to each other in front of the door. This behaviour seems to be advantageous for evacuating the room as it leads to a shorter evacuation time.

\subsection{First attempts to interpretation}

The results obtained so far suggest certain interpretations which, however, need to be substantiated by further experiments with better statistics. It is obvious from the plot of the evacuation times that increasing the group size leads to a decrease in evacuation times. The density distributions show the pupils ordered in different ways for forming groups than for pairs. A possible explanation is that the persons subordinate within the group and just follow the other group members. Because of that, there may be less conflicts between persons that meet at the door in competing for space. A person is just in competition with persons of other groups, not with own group members. Increasing the group size reduces the number of possible competi- 
tors. This reduction of conflicts may have a positive influence on the evacuation time.

When the children have to show cooperative behaviour, the evacuation is slower than without this instruction. It is a possible explanation that here the effort to stay together is larger and reduces the effort to leave the room.

\section{Summary and outlook}

We performed experiments under laboratory conditions to determine the influence of social groups on evacuations. A comparison of evacuation times between runs with different group sizes shows that increasing the group sizes lowers the evacuation time. The participants order in a different way for larger groups.

These first preliminary results have to be analyzed in more detail. The statistics need to be improved by further experiments. However, with the help of the density distributions, photographs of the finish and the data of the second school we hope to get more information from the present experiments, e.g. about the microscopic mechanisms especially close to the exit. In addition, there are some few parameters that should also be analyzed, e.g. the effect of body size and age.

Acknowledgements We dedicate this contribution in grateful memory to our friend and colleague Matthias Craesmeyer.

We thank the team from the Forschungszentrum Jülich and Wuppertal University and the students and teachers of Gymnasium Bayreuther Straße and Wilhelm-Dörpfeld-Gymnasium in Wuppertal for the help with the experiments. Financial support by the DFG under grant SCHA 636/9-1 is gratefully acknowledged.

\section{References}

1. F. Müller, A. Schadschneider, in Traffic and Granular Flow '15, ed. by V.L. Knoop, W. Daamen (Springer, 2016)

2. F. Müller, O. Wohak, A. Schadschneider, Transp. Res. Proc. 2, 168 (2014)

3. A. Mintz, The Journal of Abnormal and Social Psychology 46, 150 (1951)

4. K. Nagel, M. Schreckenberg, Journal de Physique I 2, 2221 (1992)

5. M. Boltes, A. Seyfried, B. Steffen, A. Schadschneider, in Pedestrian and Evacuation Dynamics 2008, ed. by W.W. Klingsch, C. Rogsch, A. Schadschneider, M. Schreckenberg (Springer, 2010), pp. $43-54$

6. B. Steffen, A. Seyfried, Physica A 389, 1902 (2010)

7. G. Voronoi, Journal für die reine und angewandte Mathematik 134, 198 (1908) 